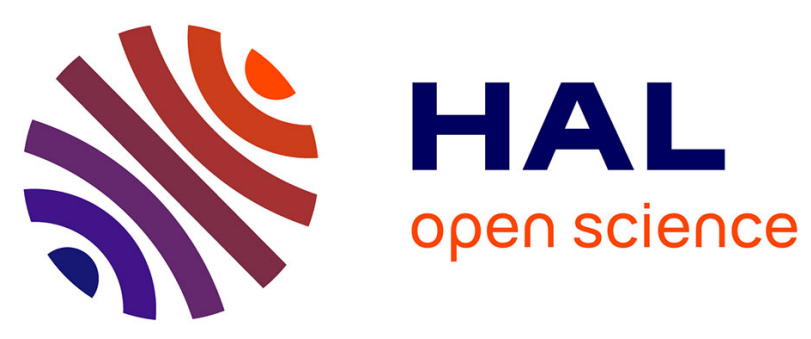

\title{
Model-free control approach for fixed-wing UAVs with uncertain parameters analysis
}

\author{
Jacson Miguel Olszanecki Barth, Jean-Philippe Condomines, Jean-Marc \\ Moschetta, Cédric Join, Michel Fliess
}

\section{- To cite this version:}

Jacson Miguel Olszanecki Barth, Jean-Philippe Condomines, Jean-Marc Moschetta, Cédric Join, Michel Fliess. Model-free control approach for fixed-wing UAVs with uncertain parameters analysis. 23rd International Conference on Methods \& Models in Automation \& Robotics, MMAR 2018, Aug 2018, Miedzyzdroje, Poland. 10.1109/MMAR.2018.8486083 . hal-01896749

\section{HAL Id: hal-01896749 \\ https://hal-enac.archives-ouvertes.fr/hal-01896749}

Submitted on 16 Oct 2018

HAL is a multi-disciplinary open access archive for the deposit and dissemination of scientific research documents, whether they are published or not. The documents may come from teaching and research institutions in France or abroad, or from public or private research centers.
L'archive ouverte pluridisciplinaire HAL, est destinée au dépôt et à la diffusion de documents scientifiques de niveau recherche, publiés ou non, émanant des établissements d'enseignement et de recherche français ou étrangers, des laboratoires publics ou privés. 
See discussions, stats, and author profiles for this publication at: https://www.researchgate.net/publication/328240831

\section{Model-Free Control Approach for Fixed-Wing UAVs with Uncertain Parameters Analysis}

Conference Paper · August 2018

DOI: 10.1109/MMAR.2018.8486083

CITATIONS

5 authors, including:

Jacson Barth

Ecole Nationale de l'Aviation Civile

3 PUBLICATIONS 2 CITATIONS

SEE PROFILE

7 Jean-Marc Moschetta

Institut Supérieur de l'Aéronautique et de l'Espace (ISAE)

125 PUBLICATIONS 631 CITATIONS

SEE PROFILE

Some of the authors of this publication are also working on these related projects:

Project Predictive control View project

Théologie de la Création View project

\section{READS}

14

Jean-Philippe Condomines

Ecole Nationale de l'Aviation Civile

29 PUBLICATIONS 42 CITATIONS

SEE PROFILE

Cédric Join

University of Lorraine

151 PUBLICATIONS 2,677 CITATIONS

SEE PROFILE 


\title{
Model-Free Control Approach for Fixed-Wing UAVs with Uncertain Parameters Analysis
}

\author{
Jacson M. O. Barth ${ }^{1}$, Jean-Philippe Condomines ${ }^{1}$, \\ Jean-Marc Moschetta ${ }^{2}$, Cédric Join ${ }^{3,5}$ and Michel Fliess ${ }^{4,5}$
}

\begin{abstract}
This paper presents first results of an innovative Model-Free Control (MFC) architecture applied to fixed-wing UAVs. MFC is an algorithm dedicated to systems with poor modeling knowledge. Indeed, the costs to derive a reliable and representative aerodynamic model for UAVs motivated the use of such a controller. By exploiting a purely numerical model, this algorithm provides an intuitive method to tune the control loop without any information about the controlled system. We propose to extend the MFC architecture to the case of fixedwing UAVs and study the MFC properties in terms of uncertain parameters. As a first result, our designed MFC architecture provides a continuous controller able to stabilize the entire flight envelope of two different fixed-wing UAVs. These results show promising adaptive perspectives and demonstrate that MFC presents robust properties for both uncertain parameters and disturbance rejection.
\end{abstract}

\section{INTRODUCTION}

The number and diversity of applications involving Micro Air Vehicles (MAVs) are extensive and have received a considerable attention in recent years. Among possible applications, different missions such as aerial imaging [1], atmospheric research [2], or even agricultural tasks [3] require effective performance in terms of endurance, range and high-speed flights which are obtained more efficiently in fixed-wing configurations. These characteristics can be improved for a specific mission profile by using aerodynamic optimization approaches which led to many innovative MAVs [4] [5] [6]. Motivated by the practical problems to find an effective control strategy which is both, simple to transpose for a new MAV and robust in terms of disturbance-rejection remains an interesting challenge for the control community. Therefore, the development of reliable and effective model-based controllers has been an important research topic (e.g., backstepping sliding mode [7], $H_{\infty}$ controller [8] [9], adaptive control [10] [11], optimal linear controllers [12]). However, these approaches require the development of an accurate model describing the aircraft dynamics that is costly and time consuming. More recently, research works

\footnotetext{
${ }^{1}$ Are with the UAV Systems Group, French Civil Aviation School, 31400 Toulouse, France. [jacson-miguel.olszanecki-barth; jeanphilippe.condomines]@enac.fr

${ }^{2}$ Is with the Department of Aerodynamics, Energetics and Propulsion, Institut Supérieur de l'Aéronautique et de l'Espace, 31400 Toulouse, France. jean-marc.moschetta@isae-supaero.fr

${ }^{3}$ Is with the CRAN (CNRS, UMR 7039), Université de Lorraine, BP 239, 54506 Vandœuvre-lès-Nancy, France. cedric.join@univ-lorraine.fr

${ }^{4}$ Is with the LIX (CNRS, UMR 7161), École polytechnique, 91128 Palaiseau, France. Michel.Fliess@polytechnique.edu

${ }^{5}$ Are with AL.I.E.N (Algèbre pour Identification \& Estimation Numérique), 7 rue Maurice Barrès, 54330 Vézelise, France. [michel.fliess; cedric.join]@alien-sas.com
}

on incremental non-linear dynamic inversion (INDI) [13] have been led and provide a less model-dependent controller that is robust for disturbance rejection. Unfortunately, INDI requires a model of actuators and test flight data to tune the control parameters. For this purpose, Model-Free Control algorithms have been developed providing a potential strategy for designing autopilots without considering any model [14] [15] [16] [17] [18]. Among them, nonlinear MFC strategy [16], has been applied in a nonlinear and strongly coupled system providing good performances in real flights with low computational costs which encourages its use in embedded systems. Whereas MFC approach can be viewed as a potential and efficient method for dealing with identification problems [19] [20]. A recent preliminary study proposed by [21] compared the MFC architecture with a model-based control for Fixed-Wing UAV with transitioning flight capabilities. This comparative study showed a better control performance obtained by the MFC approach during transition flight simulations. While recalling basic motion equations of Fixed-Wing MAVs in §II, the main contributions of this paper are therefore :

- expliciting in $\S I I I$ the theoretical equations that describe MFC architecture in the benchmarking case of the Fixed-Wing MAVs;

- studying the MAV stability in $§ I V$, for desired trajectories in forward-flight mode;

- providing new preliminary results focusing on robust properties for both uncertain parameters and disturbance rejection.

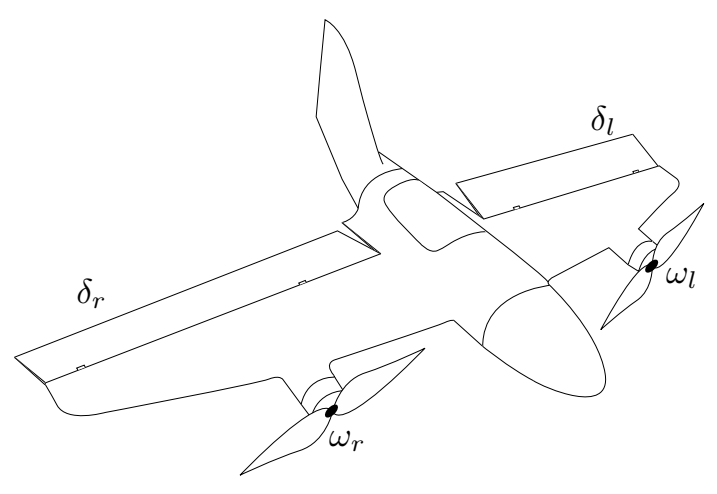

Fig. 1: A typical representation of fixed-wing MAV actuators: Propeller speeds $\left(\omega_{l}, \omega_{r}\right)$ and flap deflections $\left(\delta_{l}, \delta_{r}\right)$. 


\section{FIXED-WING MAV MODEL}

In order to tackle a wide range of innovative mini-UAVs, various flight dynamics models, in terms of assumptions and numerical techniques, therefore exist. Fixed-wing MAVs are commonly represented by non-linear equations of motion with six-Degrees-of-Freedom (DoF) : 3 DoF correspond to the translation motion $(u, v, w)$ and the 3 remaining DoF to the rotation motion $(\phi, \theta, \psi)$. Based on Newton's second law with all forces and moments expressed in the body frame, we can describe the MAV dynamics whose angular rates are denoted by $\boldsymbol{\Omega}=[p q r]^{T}$ and their resulting derived equations are given by equation (1) [22].

$$
\begin{aligned}
& \dot{p}=\frac{I_{x z}}{I_{x x}} r-q r \frac{\left(I_{z z}-I_{y y}\right)}{I_{z z}}+q p \frac{I_{x z}}{I_{x x}}+\frac{L^{A}}{I_{x x}} \\
& \dot{q}=p r \frac{I_{x z}}{I_{x x}}-\left(p^{2}-r^{2}\right) \frac{I_{x z}}{I_{y y}}+\frac{M^{A}}{I_{y y}} \\
& \dot{r}=\frac{I_{x z}}{I_{z z}} \dot{p}-p q \frac{\left(I_{y y}-I_{z z}\right)}{I_{z z}}-q r \frac{I_{x z}}{I_{z z}}+\frac{N^{A}}{I_{z z}}
\end{aligned}
$$

Conveniently, the coordinate system was chosen so that the MAV is symmetric with respect to the $x_{b} z_{b}-$ plane, thus $I_{x y}=I_{y x}=I_{z y}=I_{y z}=0$. And the inertia matrix becomes :

$$
\mathbf{I}=\left[\begin{array}{ccc}
I_{x x} & 0 & -I_{x z} \\
0 & I_{y y} & 0 \\
-I_{x z} & 0 & I_{z z}
\end{array}\right]
$$

The resulting translational equations (3) [22], correspond to the linear accelerations.

$$
\begin{aligned}
& \dot{u}=(r v-q w)+\frac{X^{A}}{m}-g \sin \theta+\frac{T_{h}}{m} \\
& \dot{v}=(p w-r u)+\frac{Y^{A}}{m}+g \cos \theta \sin \phi \\
& \dot{w}=(q u-p v)+\frac{Z^{A}}{m}+g \cos \theta \cos \phi
\end{aligned}
$$

Where $(u, v, w)$ are the linear velocities expressed in the body frame, $g$ the gravitational constant and $\phi, \theta, \psi$ the MAV attitude, respectively, roll, pitch and yaw angles. The thrust of the propellers $\left(T_{h}\right)$ which is a squared function of propeller speeds also depends of the air density $(\rho)$ and propeller characteristic, such as the diameter, etc. Aerodynamic forces $\left(X^{A}, Y^{A}, Z^{A}\right)$ and aerodynamic moments $\left(L^{A}, M^{A}, N^{A}\right)$ are subject to aerodynamic coefficients :

$$
\begin{gathered}
{\left[\begin{array}{c}
X^{A} \\
Y^{A} \\
Z^{A}
\end{array}\right]=\frac{1}{2} \rho S V^{2}\left[\begin{array}{l}
C_{X} \\
C_{Y} \\
C_{Z}
\end{array}\right]} \\
{\left[\begin{array}{c}
L^{A} \\
M^{A} \\
N^{A}
\end{array}\right]=\frac{1}{2} \rho S V^{2}\left[\begin{array}{c}
b C_{l} \\
c C_{m} \\
b C_{n}
\end{array}\right]}
\end{gathered}
$$

where $S, b, c$ are respectively, the wing area, the wingspan and the mean chord.

Remark : Aerodynamic forces can also be modelled using the $\Phi$-Theory proposed by [23].
The kinematic attitude equations (6) are used to relate the angular rates to Euler angles [22].

$$
\begin{aligned}
\dot{\phi} & =p+\tan \theta(q \sin \phi+r \cos \phi) \\
\dot{\theta} & =q \cos \phi-r \sin \phi \\
\dot{\psi} & =\sec \theta(q \sin \phi+r \cos \phi)
\end{aligned}
$$

The nonlinear state space representation corresponding to the Fixed-Wing MAV can be described in a compact form such as: $\dot{\boldsymbol{x}}=f(\boldsymbol{x}, \mathbf{u})$ and $\boldsymbol{y}=h(\boldsymbol{x}, \boldsymbol{u})$, where $\boldsymbol{x}=$ $\left(\boldsymbol{v}_{l} \boldsymbol{\omega}_{b} q\right)^{T}$ and $\boldsymbol{v}_{l}, \boldsymbol{\omega}_{b} \in \mathbb{R}^{3}, q \in \mathbb{R}^{4}$, denote respectively, vehicle velocity $(u, v, w)$ in body frame, angular velocity in body frame, and vehicle attitude represented in quaternion formulation. Control inputs $\boldsymbol{u}=\left(\omega_{l} \omega_{r} \delta_{l} \delta_{r}\right)^{T}$ are defined according to Fig. 1.

\section{MODEL-FREE CONTROL}

Model-Free Control term appears many times in the literature, but in distinct meanings from this paper. Actually, the growing importance of artificial intelligence and machine learning techniques, particularly through neural networks, has naturally been implanted into the model-free terms: see, for example [24] [25]. However, in this paper, we assume model-free control terms according to [20].

\section{A. MFC Theory}

We present briefly the main theoretical principles of some research works dealing with model-free control approach. Let's consider the following non linear state-space representation defined by :

$$
\left\{\begin{array}{l}
\dot{\mathbf{x}}=f(\mathbf{x}, \mathbf{u}) \\
\mathbf{y}=h(\mathbf{x}, \mathbf{u})
\end{array}\right.
$$

where $\mathbf{x}, \mathbf{u}, \mathbf{y}$ are the state, input and output vectors respectively. The output $\mathbf{y}$ is not directly available but rather it is observed through a noise corruption. A model for the output can be described by the following equation :

$$
y_{m}(t)=y(t)+\omega_{n}(t)
$$

where $\omega_{n}(t)$ is the observation noise. The exploitation of the MFC principles required the definition of a particular SISO model, named Ultra-Local Model, which corresponds to replace the unknown dynamic by a purely numerical model :

$$
y_{m}^{(v)}=F_{y}+\alpha \cdot u
$$

In equation (9), $v$ is the order derivative of $y_{m}, \alpha$ is a nonphysical constant parameter and is an element of $\mathbb{R}$. Moreover, the exploitation of this numerical model requires the knowledge of $F_{y}$. This quantity represents the real dynamics of the model as well as the different disturbances which could damage the output-system performances. Thus, an accurate estimation of $F$, defined as $\hat{F}$, is crucial and plays an import role in the MFC performance. Assuming that we do not have any information about the plant, its estimation can be 


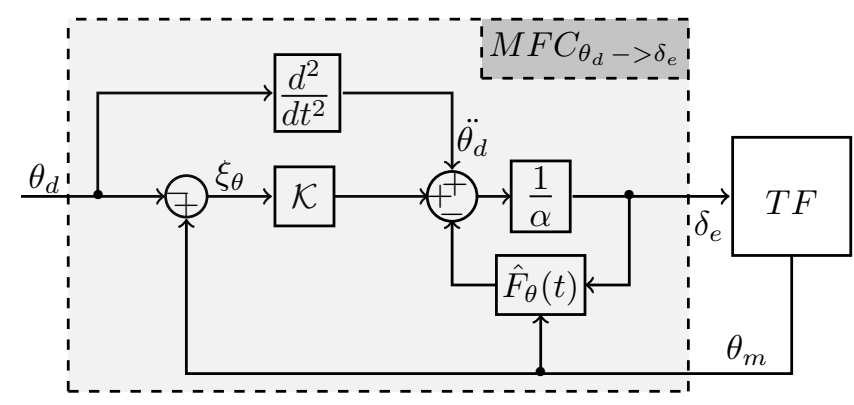

Fig. 2: Detailed Model-Free Control schema applied on equation (16). Proportional-Derivative control $\mathcal{K} . \hat{F}_{\theta}(t)$ estimator of pitch dynamic and disturbances with $\alpha$ a non-physical constant parameter.

computed directly by considering the following methodology in which we use a second-order ${ }^{1}$ Ultra-Local Model :

$$
\ddot{y}_{m}=F_{y}+\alpha \cdot u
$$

The first step is to apply the Lapace Transform in the equation (10). Referring to elementary operational calculus we transform the equation (10) to equation (11) :

$$
s^{2} Y_{m}(s)-s y_{m}(0)-\dot{y}_{m}(0)=\frac{F_{y}}{s}+\alpha U(s)
$$

Where $Y_{m}(s)$ and $U(s)$ correspond to the Laplace transforms of $y_{m}$ and $u$. By differentiating twice the previous equation we are able to rid the initial condition :

$$
2 Y_{m}(s)+4 s \frac{d Y_{m}(s)}{d s}+s^{2} \frac{d^{2} Y(s)}{d s^{2}}=\frac{2 F_{y}}{s^{3}}+\alpha \frac{d^{2} U(s)}{d s^{2}}
$$

However, $s$ in the time domain corresponds to the derivation with respect to time and it is sensitive to noise corruptions. Therefore, in order to reduce both noise and numerical computation errors on the output estimation, we replace the derivative terms by integrators $\left(\frac{1}{s}\right)$ who have robust properties with respect to noise. Thus, multiplying both sides of equation (12) by $s^{-3}$, we obtain :

$$
\frac{2 Y_{m}(s)}{s^{3}}+\frac{4}{s^{2}} \frac{d Y_{m}(s)}{d s}+\frac{1}{s} \frac{d^{2} Y(s)}{d s^{2}}=\frac{2 F_{y}}{s^{6}}+\frac{\alpha}{s^{3}} \frac{d^{2} U(s)}{d s^{2}}
$$

Equation (13) can be transferred back to the time domain employing elementary calculus and Cauchy's formula to reduce multiple integrals in a simple one :

$$
\begin{gathered}
\hat{F}_{y}=\frac{5 !}{2 T^{5}} \int_{t-T}^{t}\left[(T-\sigma)^{2}-4 \sigma(T-\sigma)+\sigma^{2}\right] y_{m}(\sigma) \\
-\left[\frac{\alpha}{2} \sigma^{2}(T-\sigma)^{2} u(\sigma)\right] d \sigma
\end{gathered}
$$

From measurements of $y_{m}$ and $u$ the unmodeled dynamic of $y$ and the disturbances $\omega_{n}$ are estimated by $\hat{F}_{y}$ which is updated for each interval of integration $[t-T, t]$. This interval corresponds to the window width of a receding horizon strategy which results in a trade-off. The idea is to choose the window width small so as to calculate the

\footnotetext{
${ }^{1}$ The same methodology can be applied to find the mathematical expression of $\hat{F}_{y}$ for a first-order Ultra-Local Model.
}

estimation within an acceptable short delay but large enough in order to preserve the low-pass filter properties whose noise attenuation of $y_{m}$. Based on such estimator it is possible to design a robust controller that estimates on-line the system dynamic from periodic measurements of $y_{m}$ and $u$. The general form of the close-loop control can be defined such as :

$$
u=\underbrace{-\frac{\hat{F}_{y}}{\alpha}}_{\text {NL Cancellation }}+\underbrace{\frac{y_{d}^{(v)}+\mathcal{K}(\xi)}{\alpha}}_{\text {Closed loop tracking }}
$$

where the quantity $\xi=y_{m}-y_{d}$ is the tracking error and $\mathcal{K}(\xi)$ is a closed loop feedback controller. We recognize in equation (15) the typical mathematical expression of a "nominal control" in the "flatness-based" control (see [26] [27] for details) in which the non-linear terms $\hat{F}_{y}$ is summed with a closed loop tracking of a reference trajectory $t \rightarrow y_{d}(t)$.

\section{B. Illustrative example}

We consider now a simple pitch angle dynamic of a given aircraft, the transfer function between the output $(\theta)$ and the elevator control input $\left(\delta_{e}\right)$ is described as follows :

$$
T F(s)=\frac{\theta(s)}{\delta_{e}(s)}=\frac{1.151 s+0.1774}{s^{3}+0.739 s^{2}+0.921 s}
$$

A second order Ultra-Local Model $(v=2)$ was chosen to estimate the pitch dynamic $(\theta)$ :

$$
\ddot{\theta}_{m}=F_{\theta}+\alpha \cdot \delta_{e}
$$

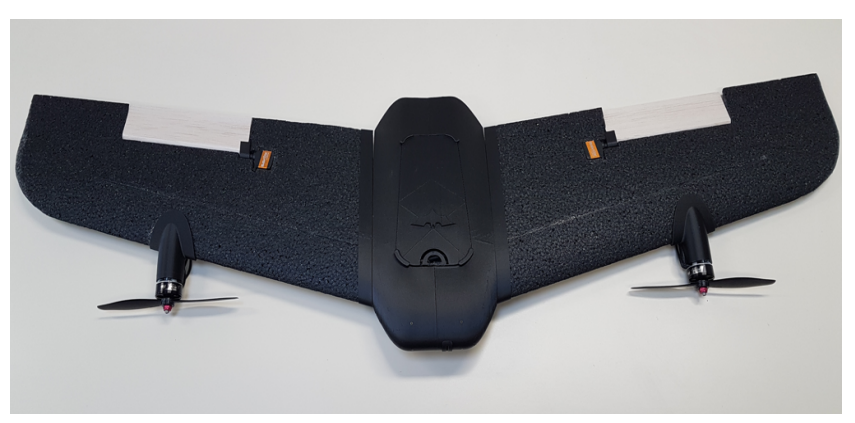

Fig. 3: Dark-Knight MAV.

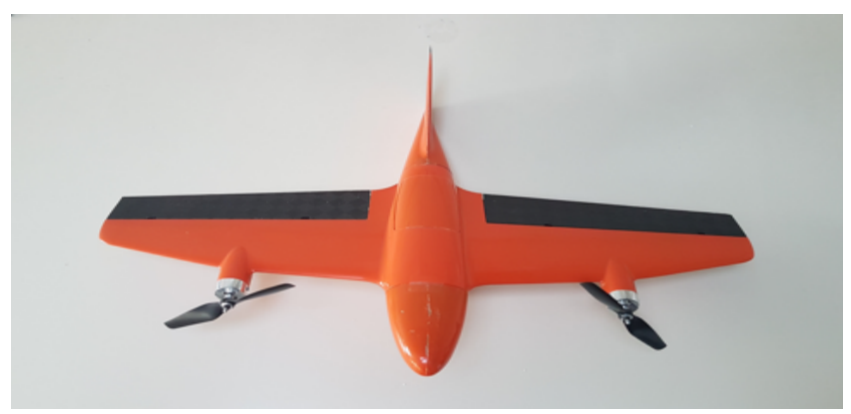

Fig. 4: Cyclone MAV. 


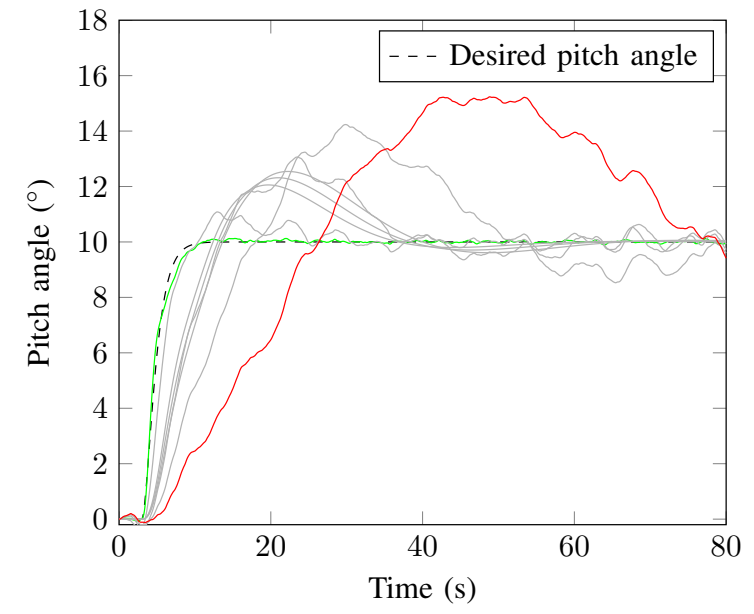

Fig. 5: Pitch angle responses with noise for different combinations of $\alpha$ and $T$ with $K_{p}=-1.5$ and $K_{d}=-2.5$.

Figure 2 shows the MFC schema which the closed-loop control can be computed by :

$$
\delta_{e}=\frac{-\hat{F}_{\theta}+\ddot{\theta}_{d}+\mathcal{K}\left(\xi_{\theta}\right)}{\alpha}
$$

Replacing (18) in (17) with $\mathcal{K}$ equal to ProportionalDerivative gains, we have :

$$
\ddot{\theta}_{m}=F_{\theta}-\hat{F}_{\theta}+\ddot{\theta}_{d}+K_{p} \xi_{\theta}+K_{d} \dot{\xi}_{\theta}
$$

It follows that theoretically, if the error between the pitch angle estimator and the real pitch angle, is approximately zero during $[t-T, t]$ :

$$
F_{\theta}-\hat{F}_{\theta} \approx 0
$$

The pitch angle and the dynamic error $\left(\xi_{\theta}\right)$ can be easily tuned by proportional and derivative gains, respectively $K_{p}$ and $K_{d}$ such that :

$$
\begin{gathered}
\ddot{\xi}_{\theta}=\ddot{\theta}_{m}-\ddot{\theta}_{d} \\
\ddot{\xi_{\theta}}-K_{p} \xi_{\theta}-K_{d} \dot{\xi}_{\theta}=0
\end{gathered}
$$

The MFC performance varies according to the following parameters: the length of the integration window $T$; The coefficient $\alpha$ that is chosen to set the same magnitude between $\theta_{m}$ and the control input $\delta_{e} . K p$ and $K d$ which are used to set the error dynamic, see Fig. 5.

Remark : It is important to emphasize that MFC algorithms have been developed to Single-Input Single-Output (SISO) systems and Fixed-Wing MAVs are Multiple-Input MultipleOutput (MIMO) systems. In our study-case, a second order Ultra-Local Model $(v=2)$ was chosen to represent each state dynamic of the MAV (attitude and velocities). Wherefore, a control architecture composed by multiple SISO MFCs, is proposed, and developed in the MFC architecture block, see Fig. 6.

\section{FLIGHT SIMULATIONS}

We now apply the control approach described in the previous section for two fixed-wing MAVs (Fig. 3 and Fig. 4) whose specifications are described in Table I. The idea is to study the MFC properties in terms of uncertain parameters. The simulation is discretized at $500 \mathrm{~Hz}$ and includes addi-

TABLE I: Fixed-Wing specifications

\begin{tabular}{lcccc}
\hline \hline Parameters & Cyclone & Dark-Knight & SI Units & $\Delta \%$ \\
\hline Mass & 0.852 & 0.586 & {$[\mathrm{Kg}]$} & 45.39 \\
$I_{x x}$ & 0.00821 & 0.00541 & {$\left[\mathrm{Kg} \mathrm{m}^{2}\right]$} & 52.30 \\
$I_{y y}$ & 0.00798 & 0.00523 & {$\left[\mathrm{Kg} \mathrm{m}^{2}\right]$} & 52.47 \\
$I_{z z}$ & 0.01641 & 0.01082 & {$\left[\mathrm{Kg} \mathrm{m}^{2}\right]$} & 51.20 \\
Propeller radius & 0.2032 & 0.1524 & {$[\mathrm{~m}]$} & 33.33 \\
Mean Chord & 0.17 & 0.175 & {$[\mathrm{~m}]$} & 2.94 \\
Wingspan & 0.88 & 1 & {$[\mathrm{~m}]$} & 13.64 \\
Wing area & 0.1496 & 0.175 & {$\left[\mathrm{~m}^{2}\right]$} & 16.98 \\
\hline \hline
\end{tabular}

tional sensor noises and state estimation errors. Also, inspired by the Dryden Wind Turbulence Model, we add wind gusts of around $4(\mathrm{~m} / \mathrm{s})$ along $\mathrm{x}$ and $\mathrm{y}$ axes to perturb the lateral and longitudinal motions. An overview of the simulation is shown in Fig. 7. The flight path describes a take-off with a constant rate of climbing fixed at $2.5(\mathrm{~m} / \mathrm{s})$, see Fig. $7 \mathrm{~d}$. Reaching a desired altitude, the rate of climbing is ordered to zero to maintain the flight level. During this part of the flight, we can analyze especially the longitudinal dynamics, such as forward speed, rate of climbing and pitch angle. At constant altitude, left-right trajectories were imposed to validate the roll and yaw control loops. Positive east-velocity defines a positive-desired roll angle and the MAV turns right, see Fig. 7c and Fig. 7e. By analogy, a rate of climb greater than zero calls for positive pitch angles (Fig. 7d and Fig. 7f) and a higher flight level will be reached. The reverse is also true, the MAV can turn left and reaches a smaller flight level with negative-desired velocities. The thrust computed by MFC can be analyzed into two parts : the nominal thrust and the differential thrust. In the first one, both propellers turn at the same speed to ensures a forward velocity around fifteen meters per second, Fig. 7b. In the second one, propellers turn at different speeds creating a moment around the $\mathrm{z}$ axis. This

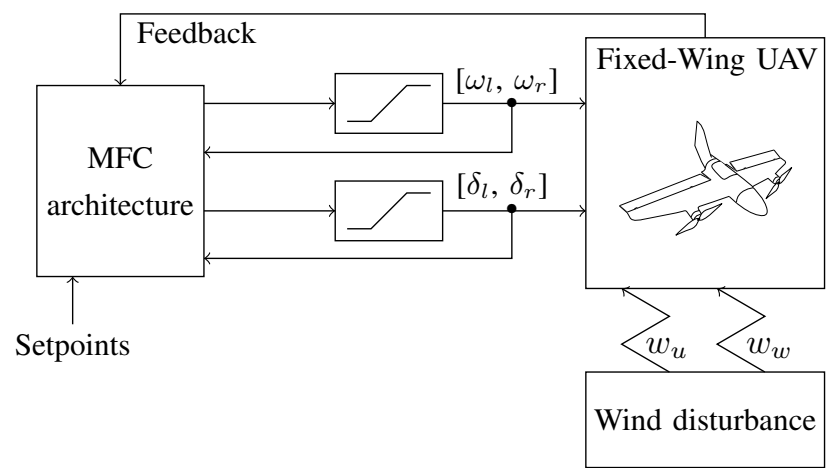

Fig. 6: MFC architecture designed for HMAVs with saturated control inputs. Propeller speeds $\left(\omega_{l}, \omega_{r}\right)$ and flap deflections $\left(\delta_{l}, \delta_{r}\right)$ are computed by means of MFC architecture block. 


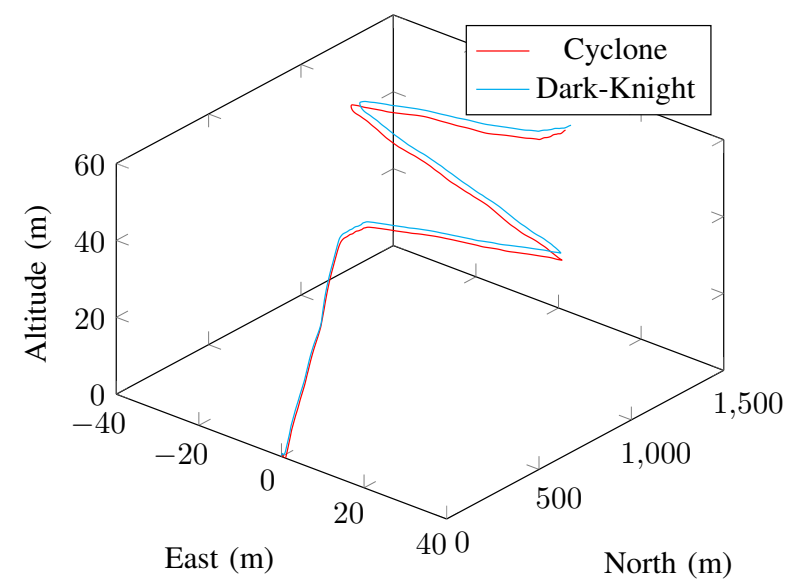

(a)

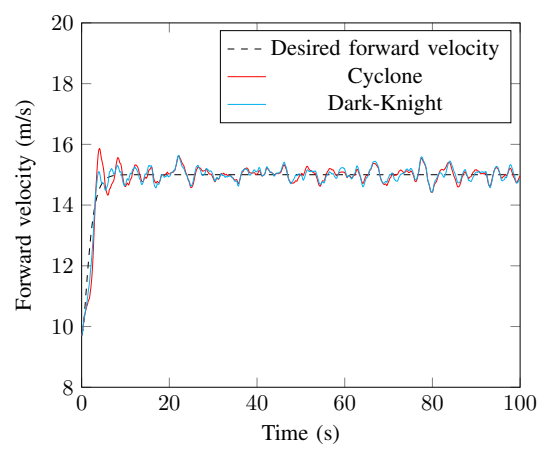

(b)

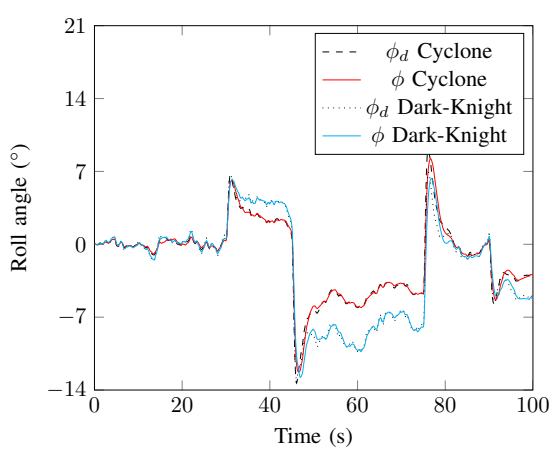

(e)

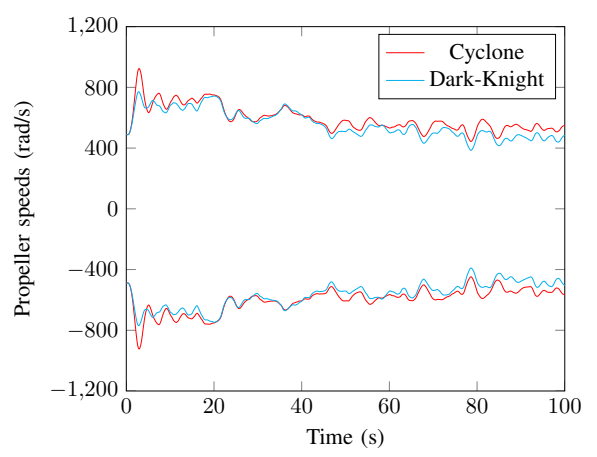

(h)

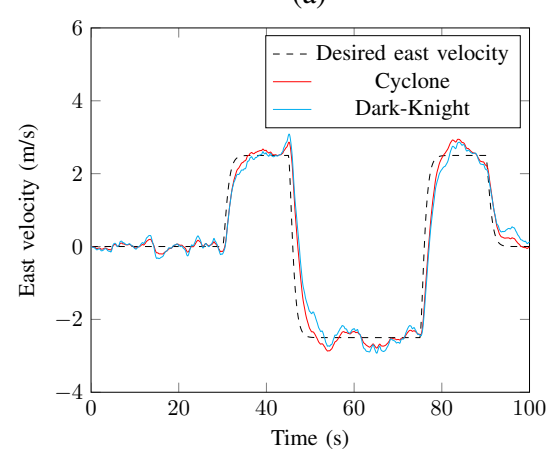

(c)

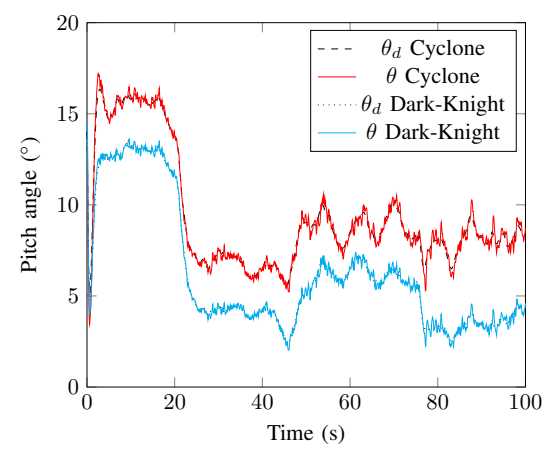

(f)

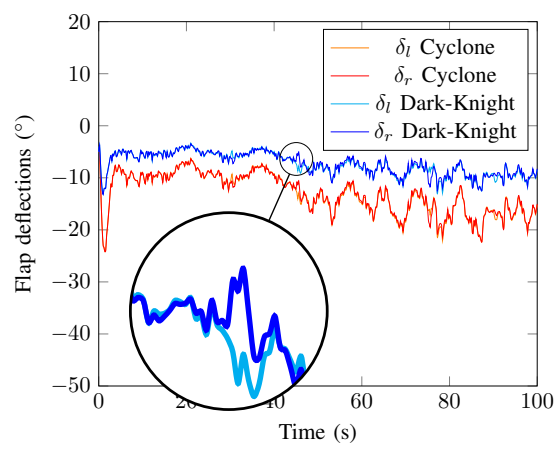

(i)

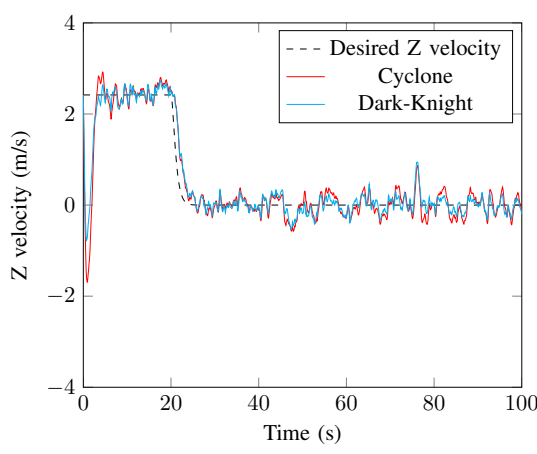

(d)

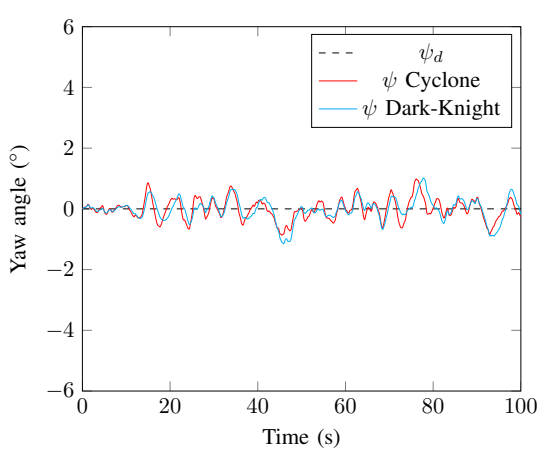

(g)

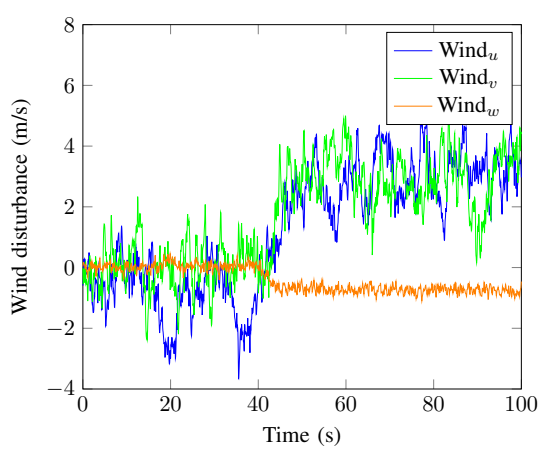

(j)

Fig. 7: Forward flight simulations. On the top : the flight path. From left to right : forward-speed, east velocity and velocity along $\mathrm{z}$ axis. Attitude in the third ligne and on the bottom : propeller speeds $\left(\omega_{l}<0\right.$ and $\left.\omega_{r}>0\right)$ due to counter-rotation sense, elevon deflections, convention negative for pitch-up $\left(\delta_{l}\right.$ and $\left.\delta_{r}\right)$ and wind disturbances. 
moment controls the yaw angle that is set to zero throughout the simulation, as shown in the Fig. $7 \mathrm{~g}$. The performance of the actuators are presented in the Fig. 7h and Fig. 7i. Cyclone flaps present greater deflection angle than for DarkKnight. This difference may be attributed to the fact that the Cyclone has a smaller wingspan. Thus, for an equivalent airspeed, the Cyclone needs a higher pitch angle to generate lift and to reach the desired rate of climbing. The zoom in the Fig. 7i (around 45 seconds), allows us to see the command which generates a negative roll moment that corresponds to a left turn. Despite windy conditions Fig. 7j, MFC ensures effective attitude stabilization and tracking velocities for both MAVs during lateral and longitudinal trajectories.

\section{CONCLUSION}

We have presented velocity and attitude control using MFC architecture for fixed-wing MAVs. The proposed approach is able to stabilize the entire flight envelope without any knowledge about the controlled MAV. First results demonstrated an effective disturbance rejection and control of unmodeled dynamics with MFC by the means of its adaptive properties.

This control architecture and MFC algorithms are being implemented in Paparazzi open-source autopilot system (cf. Paparazzi project at: https://wiki.paparazziuav. org/) and experimental flights will be presented soon.

\section{REFERENCES}

[1] Donate A. Flores, Carlos Saito and Juan A. Paredes, "Multispectral imaging of crops in the Peruvian Highlands through a fixed-wing UAV system," IEEE International Conference on Mechatronics (ICM), Churchill, VIC, Australia, pp. 399-403, February 2017.

[2] Benjamin D. Reineman, Luc Lenain and W. Kendall Melville, "The Use of Ship-Launched Fixed-Wing UAVs for Measuring the Marine Atmospheric Boundary Layer and Ocean Surface Processes," Journal of Atmospheric and Oceanic Technology, 2016.

[3] James V. Henrickson, Cameron Rogers and Han-Hsun Lu, "Infrastructure assessment with small unmanned aircraft systems," International Conference on Unmanned Aircraft Systems (ICUAS), Arlington, VA USA, pp. 933-942, June 2016.

[4] Bronz, M., Hattenberger, G. and Moschetta, J.-M, "Development of a Long Endurance Mini-UAV: ETERNITY," International Journal of Micro Air Vehicles, Volume 5 (4), pp. 261-272, 2013.

[5] Bronz, M., Condomines, J.-P and Hattenberger, G., "Development of an $18 \mathrm{~cm}$ Micro Air Vehicle : QUARK," International Micro Air Vehicle Conference and Flight Competition (IMAV), Toulouse, France, September 2013.

[6] Bronz, M., Smeur, E. J. J., de Marina, H. G., Hattenberger, G., "Development of A Fixed-Wing mini UAV with Transitioning Flight Capability," 35th AIAA Applied Aerodynamics Conference, AIAA Aviation Forum, Denver, Colorado, pp. 1-14, June 2017.

[7] Espinoza, T., Dzul, A., Lozano, R. and Parada, P., "BacksteppingSliding mode controllers applied to a fixed-wing UAV," In IEEE International Conference on Unmanned Aircraft Systems (ICUAS), Atlanta, GA, USA, pp. 95-104, May 2013.

[8] Yang, C. D. and Kung, C. C., "Nonlinear $H_{\infty}$ Flight Control of General Six-Degree-of-Freedom Motions," Journal of Guidance, Control and Dynamics, 23(2), pp. 278-288, April 2000.

[9] Ferreira, H. C., Baptista, R. S., Ishihara, J. Y. and Borges, G. A., "Disturbance rejection in a fixed wing UAV using nonlinear $H_{\infty}$ state feedback," In IEEE International Conference on Control and Automation, Santiago, Chile, pp. 386-391, December 2011.

[10] Narendra, K. S. and Balakrishnan, J., "Adaptive control using multiple models," IEEE transactions on automatic control, 42(2), pp. 171-187, February 1997.
[11] A. T. Espinoza Fraire, YangQuan Chen and A. Dzul, "Fixed-wing MAV adaptive PD control based on a modified MIT rule with slidingmode control," International Conference on Unmanned Aircraft Systems (ICUAS), Miami, FL, USA, June 2017.

[12] Teng D. Chollom1, Nkemdilim Ofodile1 and Dr. Osichinaka Ubadike, "Application Techniques of Multi-objective particle swarm optimization: Aircraft flight control," 11th International Conference on Control (UKACC), Belfast, UK, September 2016.

[13] Elisabeth S. V. D. Sman, E. J. J. Smeur, Bart Remes, C. De Wagter and Q. P. Chu, "Nonlinear Dynamic Inversion and Multihole Pressure Probes for Disturbance Rejection Control of Fixed-wing Micro Air Vehicles," International Micro Air Vehicle Competition and Conference (IMAV), Toulouse, France, pp. 111-120, 2017.

[14] Younes AI Younes, Ahmad Drak, Hassan Noura, Abdelhamid Rabhi and Ahmed EI Hajjaji, "Model-Free Control of a Quadrotor Vehicle," International Conference on Unmanned Aircraft Systems (ICUAS), Orlando, FL, USA, pp. 1126-1131, May 2014.

[15] Pérez-Arancibia, N. O., Duhamel, P.-E. J., Ma, K. Y., Wood, R. J., "Model-Free Control of a Hovering Flapping-Wing Microrobot," Journal of Intelligent \& Robotic Systems, pp. 95-111, January 2015.

[16] Aneesh N. Chand, Michihiro Kawanishi, Tatsuo Narikiyo, "Nonlinear model-free control of flapping wing flying robot using iPID," IEEE International Conference on Robotics and Automation (ICRA), Stockholm, Sweden, pp. 2930-2937, May 2016.

[17] Younes Al Younes, Ahmad Drak, Hassan Noura, Abdelhamid Rabhi and Ahmed El Hajjaji, "Robust Model-Free Control Applied to a Quadrotor UAV," Journal of Intelligent \& Robotic Systems, pp. 3752, December 2016.

[18] Haoping Wang, Xuefei Ye, Yang Tian, Gang Zheng, and Nicolai Christov, "Model-FreeBased Terminal SMC of Quadrotor Attitude and Position," IEEE Transactions on Aerospace and Electronic Systems, Vol. 52, pp. 2519-2528, October 2016.

[19] Fliess M. and Join C., "Model-free control and intelligent PID controllers: towards a possible trivialization of nonlinear control?," 15th IFAC Symposium on System Identification (SYSID), 2009.

[20] Fliess M. and Join C., "Model-free control," International Journal of Control, Taylor \& Francis, vol. 28(12), pp. 2228-2252, 2013.

[21] J. M. O. Barth, J.-P. Condomines, M. Bronz, L. R. Lustosa, J.-M. Moschetta, C. Join and M. Fliess, "Fixed-wing UAV with transitioning flight capabilities : Model-Based or Model-Free Control approach? A preliminary study," International Conference on Unmanned Aircraft Systems (ICUAS), Dallas, TX, USA, June 2018.

[22] Warren F. Phillips, "Mechanics of Flight," Second Edition, Wiley, 2009.

[23] L. R. Lustosa, F. Defay, and J.-M. Moschetta, "A global singularityless polynomial-like aerodynamics model for algorithmic flight control of tail-sitters," AIAA Journal of Guidance, Control, and Dynamics (to appear).

[24] Lillicrap, T. P., Hunt, J. J., Pritzel, A., Heess, N., Erez, T., Tassa, Y., Silver, D., and Wierstra, D., "Continuous control with deep reinforcement learning," 6th International Conference on Learning Representations, Vancouver, 2016.

[25] Radac M.-B., Precup R.E., Roman R.C., "Model-Free control performance improvement using virtual reference feedback tuning and reinforcement Q-learning," International Journal of Systems Science, September 2016.

[26] M. Mboup, C. Join and M. Fliess, "A revised look at numerical differentiation with an application to nonlinear feedback control," In 15th Mediterranean conference on Control and automation (MED'07). Athenes, Greece, July 2007.

[27] J. Zehetner, J. Reger and M. Horn, "A Derivative Estimation Toolbox based on Algebraic Methods - Theory and Practice," 2007 IEEE International Conference on Control Applications, Singapore, pp. 331336, 2007. 